\title{
Comparison of backward-scattered detection and forward-scattered detection for measuring optical force in optical tweezers
}

\author{
Yi-Jr Su and Long Hsu* \\ Institute and Department of Electrophysics, National Chiao-Tung University, 1001 Ta Hsueh Road, \\ Hsinchu, Taiwan, 30010, R.O.C
}

\begin{abstract}
Optical tweezers have become an important tool to measure forces in biology. The trapped particle displacements acquired from the position detection system are applied to calibrate trapping stiffness using power spectrum method. The near infrared light is typically used as a laser source to reduce the damage to a cell or cellular organelles and the biological objects can be held and moved by exerting piconewton forces. In force measurement, optical force strength is calculated by multiplying trapping stiffness and trapped bead displacement. Optical tweezers perform a wider range of experiments through the integration of a quadrant photodiode for position detection. Both forward-scattered detection and backward-scattered detection are the typical position detection. This study discussed both backward-scattered detection and forward-scattered detection that add a probing beam and their linear detection ranges that describe the precise position of the trapped bead. This work also discussed their linear detection ranges related to the distance between the two laser system focuses, confirming the optimum positions of the two focuses. The result indicated that the linear detection range of backward-scattered detection is longer than the forward-scattered detection. Hence, backwardscattered detection measures the longer displacement of the trapped bead in optical force measurement.
\end{abstract}

Keywords: Optical tweezers, Optical trap, Backward-scattered detection, Forward-scattered detection

\section{INTRODUCTION}

Optical tweezers ${ }^{1}$ have been extensively utilized to trap and manipulate microscopic objects. The scheme involves a continuous laser beam that is focused by a microscope objective. A trapped particle experiences a restoring force in the focal region. This effect can trap microscopic particles with an index of refraction that exceeds that of the surrounding medium. Consequently, optical tweezers are an excellent tool in biophysics. As well as trapping and manipulating particles, optical tweezers can also determine the force exerted on the trapped particles. When the optical tweezers capture a particle, the force exerted on the particle is proportional to the displacement of the particle from the center of the trap. Hence, they are very effective in measuring forces and widely adopted to investigate diffusion in cell membranes, the elasticity of cell membranes, and the mechanical properties of single molecules.

Optical tweezers perform a wider range of experiments through the integration of a quadrant photodiode (QPD) for position detection ${ }^{2,3}$. In the most common setups, the position of a trapped particle with respect to the trap center is accurately described by measuring the deflection of the forward-scattered light transmitted through the particle. The trapped particle, driven by Brownian motion, is in a harmonic potential built by the optical tweezers. Generally, these trapped particle signals from the position detection system are applied to calibrate stiffness of the optical tweezers by mean square displacement method ${ }^{4}$ or power spectrum method ${ }^{5,6}$. In force measurement, adhesive force strength is calculated by multiplying trapping stiffness and trapped bead displacement.

However, in forward-scattered detection, the cellular environment affects the transmitted laser light when measuring individual bond strength by attaching and detaching optically trapped particles to immobilized cells. The transmitted laser light is the interference between forward-scattered light from the bead and unscattered light. Many kinds of suspensions from a cellular medium influence the interference when the transmitted laser light passes through the cellular environment. Backward-scattered detection effectively reduces the cellular environment influence because the objective directly collects backward-scattered light without passing through the cellular environment. Furthermore, the

\footnotetext{
*long@cc.nctu.edu.tw
}

Optical Trapping and Optical Micromanipulation VII, edited by Kishan Dholakia, Gabriel C. Spalding, Proc. of SPIE Vol. 7762, 77623D · ( ) 2010 SPIE · CCC code: 0277-786X/10/\$18 · doi: 10.1117/12.865361 
setup of backward-scattered detection no longer requires a condenser, making it possible to combine the optical tweezers with other techniques such as the atomic force microscope. The current study discusses both backward-scattered detection and forward-scattered detection that add a probing beam and their linear detection ranges that describe the precise position of the trapped bead. This study also discusses both detections related to the linear detection range and the distance between the two laser system focuses, confirming the optimum positions of the two focuses.

\section{EXPERIMENTAL SETUP}

Figure 1 shows the experimental setup of our optical tweezers with backward-scattered detection. A fiber laser (IPG Photonics YLR-10-1064, $1064 \mathrm{~nm}$ ) was used as a light source for the trapping laser. The laser was reflected by a hot mirror (HM1) and its diameter overfilled the back aperture of the objective. A half-wave plate and a polarizing beam splitter cube were integrated to control the laser power. A reflective mirror (AM) driven with piezoelectric actuators (New Focus 8807) reflected the trapping laser and moved the optical trap along the horizontal plane. A simple telescope (L1, L2) was used to change the laser divergence that enters the objective and the axial location of the laser focus. A second telescope (L3, L4), typically in a 1:1 configuration, was used to keep the trapping power constant in the specimen plane. Because the reflective mirror and the back aperture of the objective, optically conjugate to each other, have the same diameter of the trapping laser regardless of the laser movement. The experiment steered the laser beam into a modified inverted microscope (Nikon TE2000-U) and the high numerical aperture (NA) oil-immersion objective (Nikon Plan Apo VC, 100X, NA=1.4) yielded a tight focus to trap micro-sized beads. The maximum power of the trapping laser at the back aperture of the objective was $800 \mathrm{~mW}$.

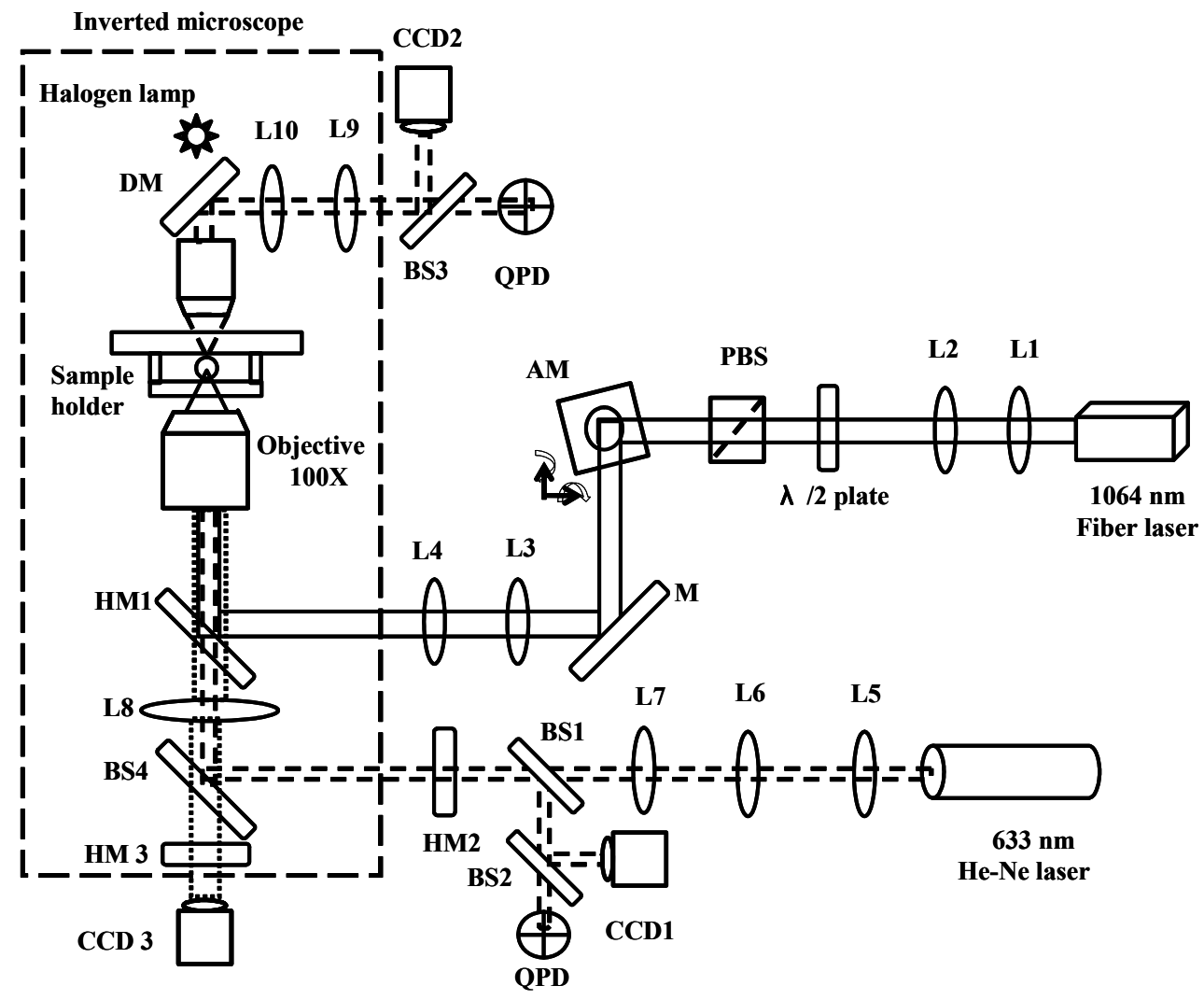

Figure 1. Schematic diagram of the optical tweezers system with backward-scattered and forward-scattered detection. Experimental setup: PBS: polarization beamsplitter cube, BS: beamsplitter, AM: gimbal-mounted mirror driven with piezo actuators, M: reflective mirror, L: lens, HM: hot mirror, QPD: quadrant photodiode, and CCD: charge coupled device camera. 
This study used the probing laser (LASOS Lasertechnik LGK 7628, $632.8 \mathrm{~nm}$, He-Ne Laser) to detect the position of a trapped micro-sized bead. A telescope (L5, L6) with micrometer position adjustment steered the focal spot of the detection beam. The laser passed through a beam splitter and then moved into the microscope via the side port. The high NA objective simultaneously collected the backward-scattered light of the probing laser from the trapped bead, and the light was imaged on a quadrant photodiode (QPD; Hamamatsu G6849) through a hot mirror (HM2) and a beam splitter (BS1). A second beam splitter (BS2) in the detection path was used to observe the backward-scattered pattern with a charge coupled device camera (CCD1).

The QPD was mounted on a dual-axis translation stage to move the QPD position so that the backward-scattered pattern could be projected on the center of the detector. The QPD converted the power of incident light to four voltages to monitor the position of the trapped micro-sized bead. The voltage difference in each direction was converted to an intensity independent position signal. The voltage signal outputs from the QPD were processed by a preamplifier and then by a main amplifier (MSR-Technik ffner) with a maximum amplification factor of 500 and a cut-off frequency of 1 $\mathrm{MHz}$. Voltage signals of the four channels were simultaneously recorded by a data acquisition card with a sampling rate of up to $10 \mathrm{MHz}$. A bright-field illumination was achieved using a halogen lamp through a condenser. The images were taken using a charge coupled device camera (CCD3) with a hot mirror (HM3) blocking infrared light. In this experiment, the $\mathrm{x}-\mathrm{y}$ position of the sample was controlled precisely based on the piezo-electrical controlled sample holder.

Both forward-scattered detection and backward-scattered detection have the same trapping setup. A condenser lens with a NA of 0.4 was added on the upper side of the sample holder to collect forward-scattered light in forward-scattered detection. The QPD was placed behind the conjugate back focal plane of the condenser lens. A beam splitter (BS3) was placed in between the condenser lens and the QPD and 50\% of the transmitted light on a camera (CCD2) for observing the forward-scattered pattern. A dichroic mirror in front of this beam splitter was used to reflect the transmitted light and allow the invisible infrared light to pass.

\section{FORCE CALIBRATION}

The trap stiffness was obtained from the position signals of the Brownian motion of the trapped particle through a twostep calibration procedure. First, calibration of the QPD was required to determine the relation between the voltage signal and the displacement of the particle with respect to the center of the optical trap. Next the signals of the Brownian motion of the trapped particle were analyzed to estimate the trap stiffness by the power spectrum method.

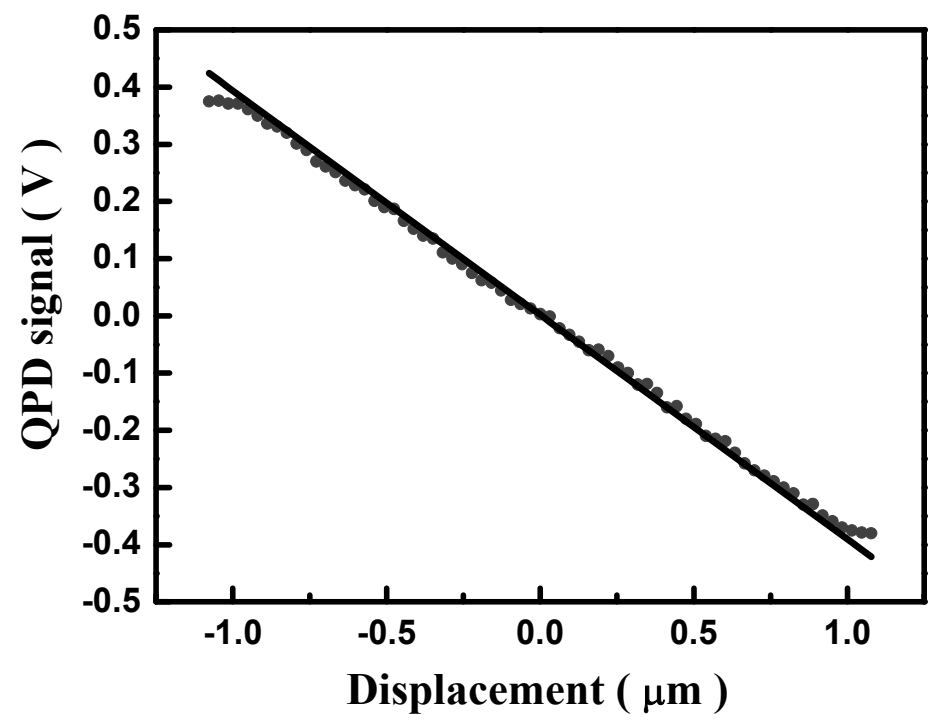

Figure 2. This diagram shows the position calibration of the quadrant photo detector. The detector responses to the trapped bead displacements are described in the gray dot and their fitting line described by the black line. 
At the first step, calibration was required to determine the relation between the voltage signal and the position of the particle. The voltage power spectrum Density (PSD) $S_{V}(f)$ is proportional to the displacement PSD $S_{x}(f)$ of the trapped particle: $S_{V}(f)=\beta^{2} S_{x}(f)$, where $\beta$ is termed the detector sensitivity and $f$ is frequency. In backward-scattered detection, the trapped bead of $3 \mu \mathrm{m}$ diameter was moved by tilting the gimbal-mounted mirror to pass through the focus of the probing laser to acquire figure 2. The figure describes the relations between the detector responses and the trapped particle positions. The QPD signals to the trapped bead displacements are described in the gray dot and their fitting line is described by the black line. This work provides a detector sensitivity of $0.394 \mathrm{~V} / \mu \mathrm{m}$.

At the second step, the PSD method was used to estimate the trap stiffness. The Brownian motion of a trapped bead near the center of the optical tweezers in one dimension is well described by the Langevin equation:

$$
\gamma x(t)^{\prime}+k x(t)=f(t)
$$

where $f(t)$ is the random thermal force, $k$ is the trapping stiffness, and $\gamma=6 \pi \eta a$ denotes the Stokes drag coefficient for a micro-sized bead of radius a moving in a fluid with viscosity $\eta$. The PSD of the trapped bead is described by a Lorentzian function:

$$
S_{x}(f)=\frac{\mathrm{K}_{B} T}{\gamma \pi^{2}\left(f_{c}^{2}+f^{2}\right)}
$$

where $K_{B}$ is Boltzmann's constant, $T$ is the absolute temperature, and $f_{c}, f_{c}=k / 2 \pi \gamma$, is the corner frequency. The PSD of the thermal force, $f(t)$, is given by $S_{f}(f)=4 \gamma k_{B} T$.

Figure 3 shows the PSD of a $3 \mu \mathrm{m}$ bead in backward-scattered detection, as shown by the gray line. The PSD was fitted to a Lorentzian function, as shown by the black line. This measurement yielded a corner frequency of $1070 \mathrm{~Hz}$ and a trap stiffness of $190 \mathrm{pN} / \mu \mathrm{m}$.

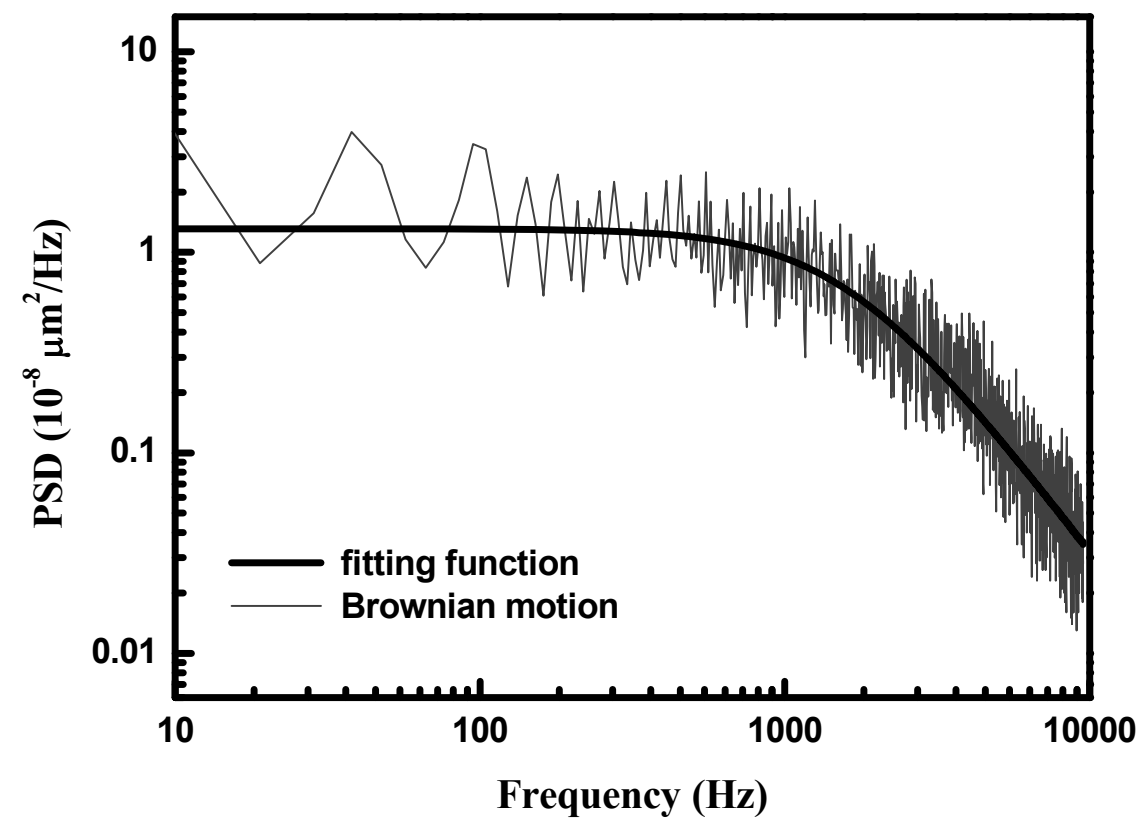

Figure 3. Power spectrum density of the thermal position fluctuations of a $3 \mu \mathrm{m}$ bead in the laser trap (gray line). The fit of a Lorentzian function to determine the corner frequency and the trapping stiffness (black line). 


\section{RESULTS AND DISCUSSION}

Figure 4 describes backward-scattered patterns and forward-scattered patterns by the position calibration of a $3 \mu \mathrm{m}$ bead. The backward-scattered patterns were recorded for the three bead displacements (a) $1.2 \mu \mathrm{m}$, (b) $0.0 \mu \mathrm{m}$, and (c) $-1.2 \mu \mathrm{m}$. The patterns not only shifted with different displacements, but also changed their shape when the optical tweezers moved the trapped bead. The forward-scattered patterns were recorded for the three bead displacements (d) $1.1 \mu \mathrm{m}$, (e) $0.0 \mu \mathrm{m}$, and (f) $-1.1 \mu \mathrm{m}$. These patterns differ from backward-scattered patterns and also precisely describe the bead's position by interference variation.

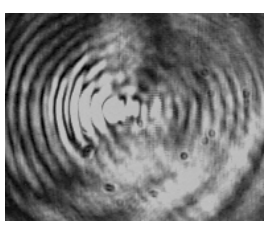

( a )

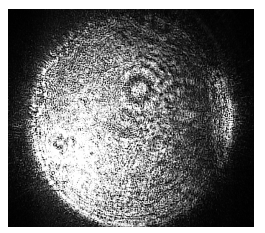

(d)

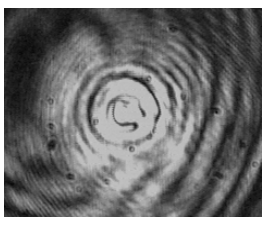

( b )

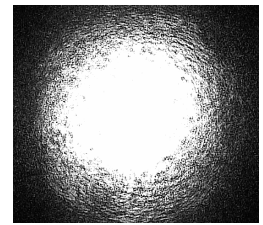

( e )

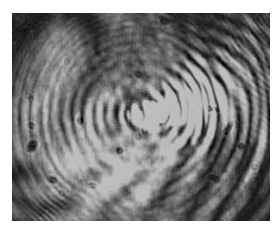

( c)

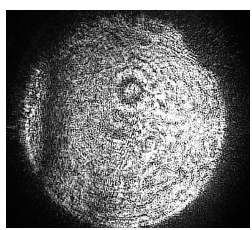

( f)

Figure 4. (a), (b) and (c) show the patterns of backward-scattered detection, and (d), (e) and (f) show the patterns of forward-scattered detection.

To precisely describe the position of the trapped bead, it is necessary to define a linear detection range where the trapped bead displacement is proportional to detector response. Using figure 2 from the position calibration of the quadrant photo detector, figure 5 shows the difference between the detector response and the fitting line, divided by the magnitude of the fitting line at $1.0 \mu \mathrm{m}$, represented an error percentage. We determined the linear detection range where the deviation was less than $5 \%$ and the linear detection range of the $3 \mu \mathrm{m}$ bead was $1.97 \mu \mathrm{m}$ in backward-scattered detection.

Under the same method discussed above, figure 6 indicates the linear detection ranges of these two detections with the vertical differences, $\mathrm{z}$, and $\mathrm{z}$ used to describe the vertical difference of two focuses between the probing laser and the trapping laser. Along the axial direction of trapping laser, $\mathrm{z}$ was positive when the focus of the probing laser was in front of the focus of the trapping laser. When the focus of the trapping laser was in front of the focus of the probing laser, $\mathrm{z}$ was negative. When these two focuses overlapped each other, $\mathrm{z}$ was zero. The linear detection range of backwardscattered detection is labeled with a solid triangle and forward-scattered detection is labeled with an inverted solid triangle. We fixed the axial location of the focus of the trapping laser in the experiment and then $\mathrm{z}$ depended on the telescope spacing that the probing laser passed through. The relation between the telescope spacing and $\mathrm{z}$ roughly was determined by ray tracing in the probing beam system. Moreover, the sample stage was finely tuned to confirm the position of the focus of the probing laser by tracing the image of the laser focus. Figure 6 indicates that both backwardscattered detection and forward-scattered detection have a maximum linear detection range. The maximum linear detection range of backward-scattered light is $1.97 \mu \mathrm{m}$ at $\mathrm{z}=2 \mu \mathrm{m}$ and forward scattered light is $1.74 \mu \mathrm{m}$ at $\mathrm{z}=-2 \mu \mathrm{m}$. The two focus positions of the probing laser, symmetrically focused on the trapping laser, provide maximum linear detection ranges. The maximum linear detection range of backward-scattered detection is longer than the forwardscattered one. 


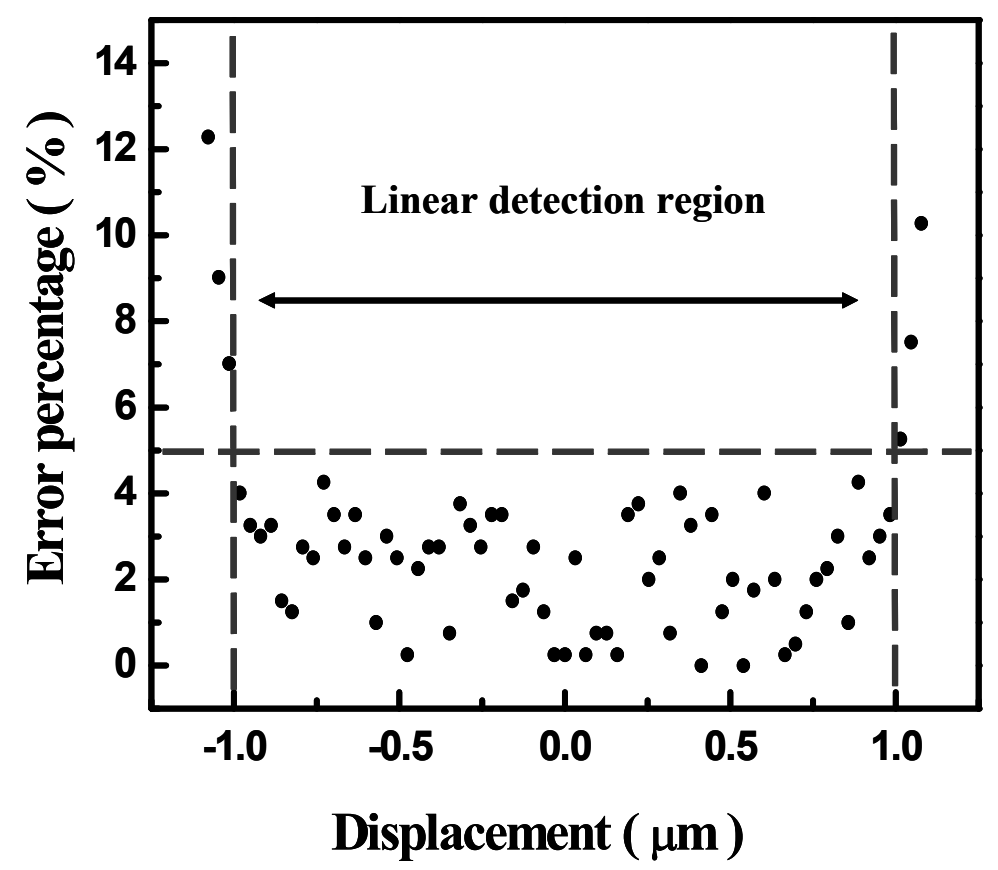

Figure 5. The diagram uses to determine the linear detection range where the deviation was less than 5\%.

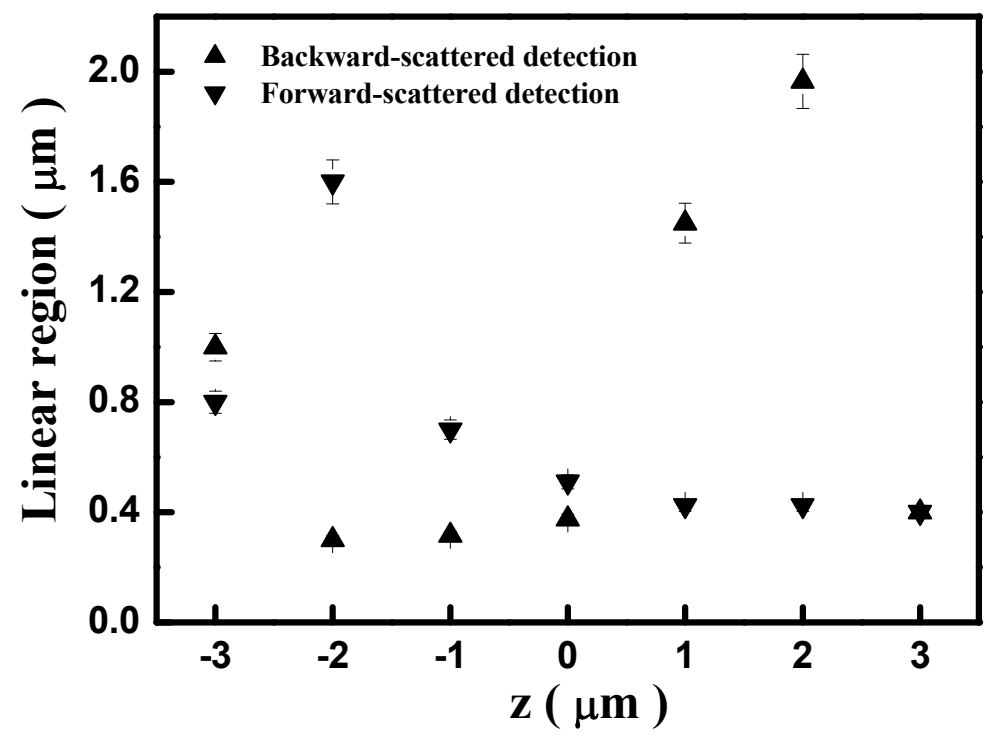

Figure 6. Comparison of the linear detection range between backward-scattered detection (solid triangle) and forwardscattered detection (inverted solid triangle). 


\section{CONCLUSIONS}

This study discusses both backward-scattered detection and forward-scattered detection to find the longest distance linear detection range for a $3 \mu \mathrm{m}$ bead. The result indicates that the linear detection range of backward-scattered detection is longer than the forward-scattered detection. This study also discusses both detections related to the linear detection range and the distance between the two laser system focuses, confirming the optimum positions of the two focuses. As discussed above, backward-scattered detection provides the longer detection range, $1.97 \mu \mathrm{m}$, at $\mathrm{z}=2 \mu \mathrm{m}$. Hence, backward-scattered detection measures the longer displacement of the trapped bead in optical force measurement.

\section{ACKNOWLEDGEMENT}

The authors would like to thank the National Science Council of the Republic of China, Taiwan, for financially supporting this research under Contract No. NSC98-2221-E-009-029

\section{REFERENCES}

[1] M. J. Lang and S. M. Block, "Resource Letter: LBOT-1: Laser-based optical tweezers," Am. J. Phys. Papers 71, 201-215 (2003).

[2] A. Rohrbach and E. H. K. Stelzer, "Three-dimensional position detection of optically trapped dielectric particles," J. Appl. Phys. Papers 91, 5474-5488 (2002).

[3] J.K. Dreyer, K. Berg-Sørensen, and L. Oddershede, "Improved Axial Position Detection in Optical Tweezers Measurements," Appl. Opt. Papers 43, 1991-1995 (2004).

[4] K. C. Neuman and S. M. Block, "Optical trapping," Rev. Sci. Instrum. Papers 75, 2787- 2809 (2004).

[5] K. Svoboda and S. M. Block, " Biological applications of optical forces," Annu. Rev. Biophys. Biomol. Struct. Papers 23, 247-285 (1994).

[6] M. W. Allersma, F. Gittes, M. J. deCastro, R. J. Stewart, and C. F. Schmidt, " Two-dimensional tracking of ncd motility by back focal plane interferometry," Biophys. J. Papers 74, 1074-1085 (1998).

[7] J. H. G. Huisstede, K. O. van der Werf, M. L. Bennink, and V. Subramaniam, "Force detection in optical tweezers using backscattered light," Opt. Express. Papers 13, 1113-1123 (2005). 